\title{
FACTORS AFFECTING SYNONYMOUS CODON USAGE OF HOUSEKEEPING GENES IN DROSOPHILA MELANOGASTER
}

\author{
Yi WeN Lu+ and TAI SHENG CHIU+* \\ Department of Life Science, National Taiwan University, No. 1, Sec. 4, Roosevelt Rd., Taipei 10617, \\ Taiwan
}

(Received: July 17, 2017; accepted: January 9, 2018)

\begin{abstract}
Housekeeping genes (HK genes) are required for cell survival and the maintenance of basic cellular functions. The investigation of factors affecting codon usage patterns in HK genes of insects can help in understanding the molecular evolution of insects and aid the development of insect pest management strategies. In this study, we employed bioinformatics approaches to analyze the codon usage bias (CUB) of HK genes in the insect model organism, Drosophila melanogaster. A comparison of CUB between $1107 \mathrm{HK}$ genes and 1084 high tissue specificity genes suggested that HK genes have higher CUB in D. melanogaster. In addition, we found that CUB inversely correlates with the non-synonymous substitution rate of HK genes. Therefore, we attempted to identify the factors that potentially influence the codon usage pattern of HK genes. Our results suggest that mutation pressure and natural selection highly correlate with CUB in the HK genes of D. melanogaster and that two topological properties of HK proteins (proportion of protein interacting length and protein connectivity) also correlate with CUB in the HK genes of D. melanogaster. This study provides insight into CUB in the HK genes of D. melanogaster, and the results can support future investigations of potential applications in agricultural and biomedical field
\end{abstract}

Keywords: Housekeeping gene - codon usage bias - mutation bias - translational selection - protein topology

\section{INTRODUCTION}

In the protein synthesis process, 61 codons code for only 20 amino acids. Multiple codons that code for the same amino acid are called synonymous codons. Synonymous codons are not used at the same frequency in several organisms, existing from prokaryotes to eukaryotes $[11,17]$. This phenomenon is known as codon usage bias (CUB). CUB varies among different species and among genes in the same genome [35]. Additionally, evidences have been accumulated that some genes with slow-evolving nonsynonymous sites have higher CUB [37]. Many factors have been proposed to be related to CUB, including base compositional mutation bias [21], gene expression level [36], gene length [25], codon-anticodon binding [28], tRNA abundance [10], protein structure [18], and so on. Although the exact mechanisms of CUB remain

\footnotetext{
*Corresponding author; e-mail address: tschiu@ntu.edu.tw
}

+The authors contributed equally to this work. 
unclear, mutation bias and natural selection are two possible candidates in many species [32]. The mutation explanation for CUB is that the non-randomness of mutational patterns affects the usage frequencies of synonymous codons (e.g., GC-rich organisms use more G- or C-ending codons). According to the natural selection explanation, CUB is caused by selection to increase the efficiency and/or accuracy of translation (such as translation selection, some highly expressed genes have higher CUB than do lowly expressed genes). In some organisms, CUB is shaped by both mutation bias and natural selection [32]. In addition to research on the influences of translational selection and mutation bias on codon usage, research on the role of protein structure in synonymous codon usage has surged in recent years [6]. In yeast proteins, $\beta$-sheets are encoded by coding sequences that are enriched in optimal codons [31]. Previous cross-taxon analyses have suggested that protein buried sites and structurally important sites are associated with optimal codons [50]. In addition, early studies reported that the proportion of protein interacting length and protein connectivity both correlate with protein evolution $[15,33]$. However, the correlations between these two topological properties and CUB are unclear.

Housekeeping genes (HK genes) are constitutively expressed in various tissues and are involved in the maintenance of cellular functions [4]. The study of synonymous codon usage in HK genes of insects can provide helpful information about the molecular evolutionary mechanisms of insects and facilitate the development of effective control strategies for insect pests to reduce insect-borne diseases and agricultural losses. In the insect model animal, D. melanogaster, the relationships between CUB and HK genes and the underlying mechanisms remain unclear. The effect of CUB on the evolution of HK genes has not been investigated in detail. In addition, few efforts have been made to explore whether any relationship exists between each of four factors (translational selection, mutation bias and two topological properties of HK proteins) and synonymous codon usage in HK genes of $D$. melanogaster. The aims of this study are to investigate the synonymous codon usage of HK genes in D. melanogaster and to assess the impact of synonymous codon usage on the evolution of HK genes and evaluate possible factors related to synonymous codon usage in HK genes of D. melanogaster. Published gene expression data concerning $13 \mathrm{D}$. melanogaster tissues were used to calculate tissue specificity and identify 1,107 HK genes and 1,084 high tissue specificity genes (HTS genes) for further synonymous codon usage analyses. The results of this study may help shed some light on the possible factors influencing synonymous codon usage in the HK genes of D. melanogaster.

\section{MATERIAL AND METHODS}

\section{Gene expression data}

Gene expression data of adult D. melanogaster were acquired from the FlyAtlas database (http://flyatlas.gla.ac.uk/) [34]. A total of 18,880 probe sets covered 13,250 D. melanogaster genes. To prevent errors associated with re-counting, we eliminated 
genes that were detected by the same probe or more than one probe set. The Presence/ Absence calls of the gene expression data were calculated according to the Affymetrix Microarray Suite (MAS) version 5.0 algorithm with the default parameters [19]. Genes were listed as "present" if statistically significant expression was detected in at least three of four replicates. Finally, we obtained 11,166 unambiguous genes with expression data. We used the gene expression data of 13 D. melanogaster tissues in this paper (brain, thoracico-abdominal ganglion, salivary gland, crop, midgut, hindgut, tubule, fat body, ovary, testis, accessory glands, virgin spermatheca, and heart). To compare the expression intensity of each gene, we calculated the mean expression intensity of each gene across the 13 tissues for analyses.

\section{Tissue specificity}

The tissue specificity index $\tau[44]$ is defined as

$$
\tau=\frac{\sum_{j=1}^{n}\left(1-\left[\frac{\log _{2} S(i . j)}{\log _{2} S(i, \max )}\right]\right)}{n-1}
$$

Where " $n$ " is the number of $D$. melanogaster tissues examined and $S(i, \max )$ is the highest expression intensity of gene $i$ across all of the examined tissues. If a gene is equally expressed in all tissues, the index is zero. However, if a gene is expressed in only one or a few tissues, the index is close to one. The genes obtained from the FlyAtlas database were sorted by tissue specificity index, and $10 \%$ of genes from each end of the list were considered. The genes with high tissue specificity (top 10\%) were defined as HTS genes. Genes that were expressed in every tissue with low tissue specificity (bottom 10\%) were defined as HK genes. Based on the above criteria, we obtained 1,107 HK genes and 1,084 HTS genes.

\section{Codon usage bias analysis}

Codon bias index (CBI) was also used to measure the usage of optimal codons in this study [1]. If a gene has extreme codon bias, the value of CBI will equal 1. If a gene shows random codon usage, the value of CBI will equal 0 .

Effective number of codons (ENC) was used to examine the codon usage bias in this study [42]. The value of ENC lies in the range of 20 to 61. If only one codon is used for each amino acid, the value of ENC is 20 . If all synonymous codons are equally used for each amino acid, the value of ENC is 61. 


\section{Nucleotide composition}

The $\mathrm{GC}$ and $\mathrm{GC}_{3}$ contents of the genes were computed via DnaSP v5 [23]. The $\mathrm{GC}_{3}$ content (the frequency of codons ending in $\mathrm{G}$ or $\mathrm{C}$ excluding Met, Trp and stop codons) is used to measure the extent of base composition bias [49].

\section{Percentage of protein interacting length}

The ratio of protein interacting domain length was calculated from following formula:

$$
\text { Percentage of protein interacting length }=\frac{\text { sum of interacting domain length }}{\text { protein length }} .
$$

Protein domain data were retrieved from the PFAM database (http://pfam.xfam.org/) [14]. The following cutoff values were used for domain assignment [20]: (1) matched sequence length $>80 \%$ of domain length; (2) e-value of alignment $<1.0 \times 10^{-4}$; (3) domain length $>12$ amino acids. Protein length data were retrieved from the UniProt database (http://www.uniprot.org/) [2].

\section{Protein-protein interaction data}

The protein-protein interaction data of D. melanogaster proteins used in this study were obtained from the IntAct database (http://www.ebi.ac.uk/intact/) [29]. To avoid false positives in the identification of protein interactions, we used two-hybrid experiment data of the protein-protein interactions from the IntAct database.

\section{Estimation of nonsynonymous substitution rates}

The coding sequences of $D$. melanogaster HK and HTS genes were obtained from FlyBase [7]. The orthologous gene sequences of D. melanogaster HK and HTS genes in D. yakuba were retrieved from OrthoDB [40]. The split between D. melanogaster and D. yakuba occurred approximately 12.8 MYA (million years ago) [38]. D. melanogaster - D. yakuba orthologous gene pairs were used for estimating the rates of nonsynonymous nucleotide substitution [24]. Nonsynonymous substitution rates were estimated based on the nucleotide alignments of each gene by using the maximum likelihood method implemented with the PAML package $[45,46]$. 


\section{Statistical analyses}

Pairwise comparisons of variables were performed with the nonparametric MannWhitney U-test. The nonparametric Spearman rank correlation test was used to assess the degree of association between two variables. SPSS software (version 22.0) was used to perform all statistical analyses.

\section{RESULTS AND DISCUSSION}

\section{Codon bias differences between HK and HTS genes}

We used ENC and CBI to examine the extent of CUB in D. melanogaster HK and HTS genes. Differences in CUB between HK and HTS genes were analyzed using the nonparametric Mann-Whitney U-test. Table 1 presents the means of ENC and CBI for HK and HTS genes. The mean CBI of HK genes was higher than that of HTS genes, and the mean ENC of HK genes was lower than that of HTS genes. The results indicated that HK genes had higher CUB than did HTS genes. We also calculated the means of $\mathrm{CBI}$ and $\mathrm{ENC}$ for 1100 genes from the middle of the tissue specificity index list. The mean CBI and ENC for this middle group were between the CUB index values of HK and HTS genes (Table 1). Why do the evolutionary forces shape the codon usage patterns of HK genes? Carlini and Stephan [5] substituted six and ten optimal codons for Leu with unpreferred codons in the Drosophila Adh gene and observed $19 \%$ and $24 \%$ decreases in $\mathrm{ADH}$ protein production, respectively. Fath et al. [13] investigated the effect of codon optimization on the protein expression levels of 50 genes representing five classes of human proteins (transcription factors, ribosomal and polymerase subunits, protein kinases, membrane proteins and immunomodulators) in Human Embryonic Kidney $293 \mathrm{~T}$ cells. They observed that $86 \%$ of the optimized constructs increased their protein expression levels. These studies revealed that CUB plays an important role in protein biosynthesis. HK genes are expressed in various tissues and play essential roles in the maintenance of cellular functions, whereas HTS genes are expressed only in one or a few tissues. Evolutionary forces might shape the codon usage patterns of HK genes, thereby contributing to the stable and sufficient biosynthesis of HK proteins for the maintenance of basic functionality in most functioning cells. Manipulation of the preferred codons of insect $\mathrm{HK}$ genes might affect the expression of insect HK proteins and might be useful for managing insect pest populations.

\section{$G C_{3}$ content and gene expression level correlate with the $C U B$ of HK genes}

HK genes have higher CUB than do HTS genes. Which factors might contribute to the higher CUB in HK genes? We hypothesized that translational selection and muta- 
tional bias correlate with CUB in HK genes of D. melanogaster. To test this hypothesis, we performed correlation analyses of CUB and these two factors. We first compared these two factors between HK and HTS genes by performing nonparametric Mann-Whitney U-tests. The mean GC content was higher for HK genes than for HTS genes (Table 1). Due to the higher GC content, G- or C-ending codons are expected to predominate in $\mathrm{HK}$ genes. We found that the $\mathrm{GC}_{3}$ content of $\mathrm{HK}$ genes correlated with the GC content of HK genes (correlation coefficient $\rho=0.774, p$ value $\left.=2.221 \times 10^{-195}\right)$. The mean $\mathrm{GC}_{3}$ content was higher for the $\mathrm{HK}$ genes than for the HTS genes (Table 1). When comparing expression level between HK and HTS genes, we found that the mean expression level was higher for the HK genes than for the HTS genes (Table 1).

Table 1

Average values of Effective number of codons (ENC), Codon bias index (CBI), GC content, $\mathrm{GC}_{3}$ content and expression level for HK and HTS genes

\begin{tabular}{|l|c|c|c|}
\hline \multicolumn{1}{|c|}{ CUB index } & HK & HTS & p value \\
\hline CBI & $0.457(0.391)$ & 0.370 & $2.588 \times 10^{-45}$ \\
\hline ENC & $46.001(49.975)$ & 51.670 & $5.248 \times 10^{-75}$ \\
\hline GC content & $55 \%$ & $51.5 \%$ & $2.622 \times 10^{-58}$ \\
\hline GC $_{3}$ content & $68 \%$ & $59.4 \%$ & $3.679 \times 10^{-78}$ \\
\hline Expression level & 804.550 & 118.183 & $2.593 \times 10^{-213}$ \\
\hline
\end{tabular}

*Values in parentheses are the mean CBI and ENC for middle group of the tissue specificity index list. P values (two tailed nonparametric Mann-Whitney U-test) show significant differences between above properties for HK gene group and HTS gene group.

The results indicate that $\mathrm{HK}$ genes have higher $\mathrm{GC}_{3}$ content and expression level than do HTS genes. Next, we conducted correlation analyses of CUB and these two factors. Table 2 and Fig. $1 \mathrm{~A}-\mathrm{B}$ show that $\mathrm{CBI}$ and ENC strongly correlate with $\mathrm{GC}_{3}$ content, suggesting that mutational bias might play an important role in the CUB of HK genes. The bias for cytosine and guanine at the third position in preferred codons has also been reported previously. Vicario et al. [39] analyzed CUB among 12 species of Drosophila genomes and found that $\mathrm{GC}_{3}$ codons were preferentially used in 11 species (i.e., all except $D$. willistoni). Previous studies reported that genes with high $\mathrm{GC}_{3}$ (or high $\mathrm{GC}$ ) content differ from those with low $\mathrm{GC}_{3}$ (or low $\mathrm{GC}$ ) content at transcription stage. Kudla et al. [22] compared the expressions of genes with different $\mathrm{GC}_{3}$ contents (human HSPA1A $\mathrm{GC}_{3}=92 \%$ and HSPA8 $\mathrm{GC}_{3}=46 \%$ ). Although the coding regions and corresponding protein sequences of these two genes are similar, the high- $\mathrm{GC}_{3}$ gene exhibited an up to 10 -fold greater protein level relative to the expression level of the low- $\mathrm{GC}_{3}$ gene. A series of experiments demonstrated that this difference was due to increasing the steady-state mRNA level of high- $\mathrm{GC}_{3}$ gene. Newman et al. [27] investigated differences in codon bias and GC content in the expression of mammalian Toll-like receptor7 genes $(T l r 7)$. They found that suboptimal codon bias (low GC content) can limit the transcription of $T l r 7$ and codon opti- 
mization (higher GC content) of Tlr7 increases the rate of transcription. Zamft et al. [47] reported that RNA polymerases II of S. cerevisiae pause for shorter periods and less frequently on GC-rich templates than they do on AT-rich templates. Mutational pressure due to GC compositional constraints might drive the evolution of synonymous codon usage in HK genes to maintain stable and efficient transcription.

To explore the relationship between CUB and gene expression level, we performed correlation analyses. As shown in Table 2 and Fig. 1C-D, CBI and ENC significantly correlate with gene expression level, indicating that gene expression level might be an important factor shaping codon usage in HK genes. A correlation between gene expression level and CUB was also observed in a previous Expressed Sequence Tag
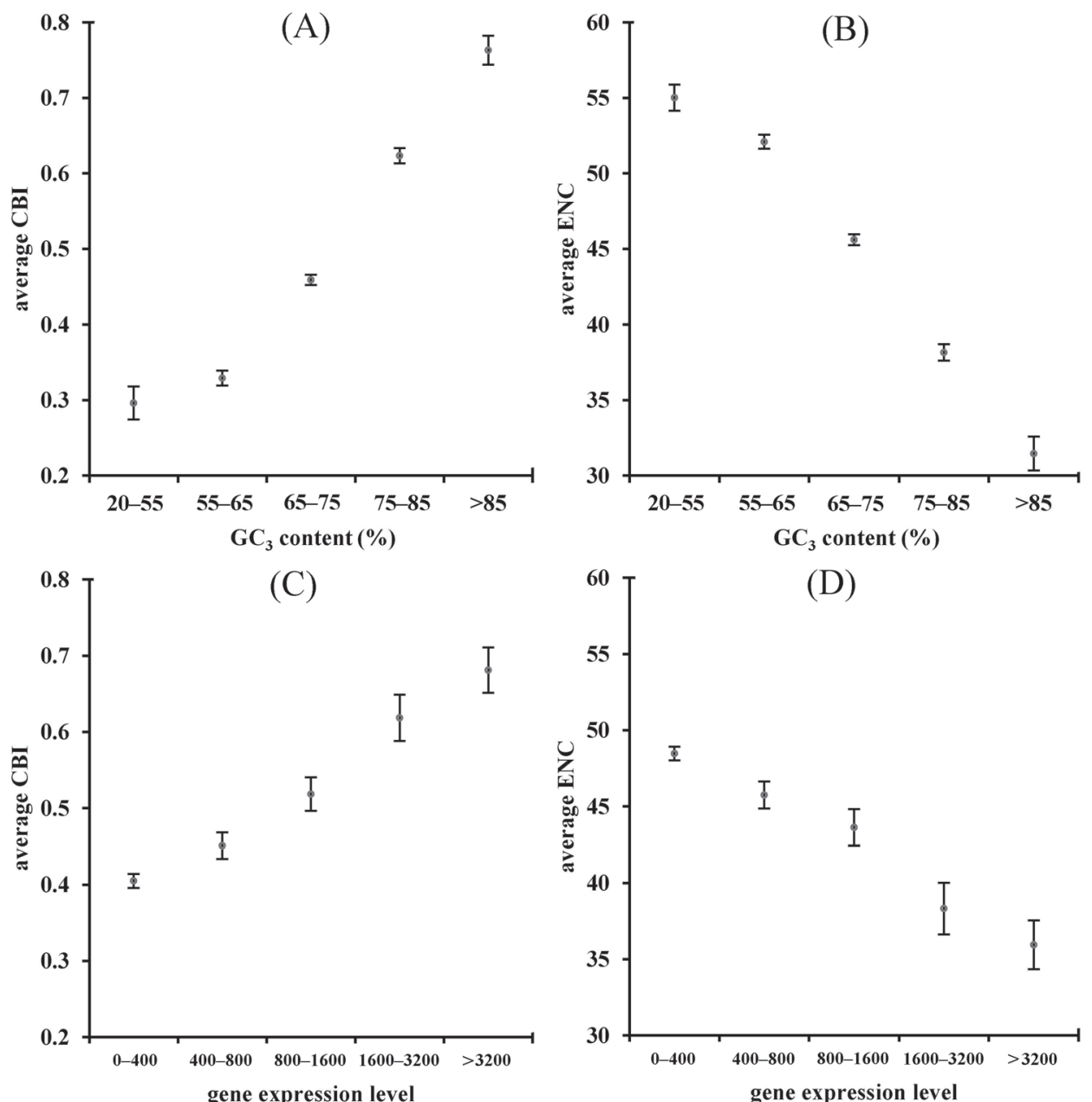

Fig. 1. Scatter Diagrams show correlation between (A) $\mathrm{CBI}$ and $\mathrm{GC}_{3}$ content (B) $\mathrm{ENC}$ and $\mathrm{GC}_{3}$ content (C) $\mathrm{CBI}$ and gene expression level (D) ENC and gene expression level in HK genes. The error bar shows $95 \%$ confidence interval of the mean 
Table 2

Spearman's rank correlation analyses between factors and CUB in HK genes of D. melanogaster

\begin{tabular}{|l|c|c|c|c|}
\hline \multirow{2}{*}{ Factor } & \multicolumn{2}{c|}{ CBI } & \multicolumn{2}{c|}{ ENC } \\
\cline { 2 - 5 } & $\rho$ & p value & $\rho$ & $\mathrm{p}$ value \\
\hline $\mathrm{GC}_{3}$ & 0.777 & $6.227 \times 10^{-224}$ & -0.774 & $5.187 \times 10^{-221}$ \\
\hline Expression level & 0.534 & $1.358 \times 10^{-82}$ & -0.468 & $3.621 \times 10^{-61}$ \\
\hline $\begin{array}{l}\text { Percentage of protein } \\
\text { interacting length }\end{array}$ & 0.319 & $1.281 \times 10^{-27}$ & -0.242 & $3.201 \times 10^{-16}$ \\
\hline Protein connectivity & 0.172 & $9.249 \times 10^{-9}$ & -0.193 & $9.541 \times 10^{-11}$ \\
\hline
\end{tabular}

Spearman's rank correlation coefficients $(\rho)$ are shown and p values are considered statistically significant.

(EST) analysis of 1,550 D. melanogaster genes [12]. Researchers have suggested that highly expressed genes are under translational selection to use the optimal codons [32]. Two hypotheses have been proposed to explain the selective forces shaping codon usage in highly expressed genes [6]. The translational efficiency hypothesis states that preferred codons are under selection in highly expressed genes because of their faster translation rate. A recent study for monitoring translation in real time in mammalian cells showed that the translation rate of codon-optimized mRNAs is faster (4.9 codons per second) than that of non-optimized mRNAs (3.1 codons per second) [43]. A cycloheximide-free ribosome profiling experiment in yeast reported that ribosome dwell times are shorter at preferred codons for abundant tRNAs [41]. Translational selection might affect the codon usage patterns of $\mathrm{HK}$ genes, leading to more efficient protein production for cells. The translational accuracy hypothesis states that natural selection biases codon usage to enhance the accuracy of translation. Drummond and Wilke [9] found that optimal codons in highly expressed genes have lower probabilities of mistranslation than nonoptimal codons in these genes do in numerous taxonomic groups. They proposed a model in which selection against toxicity from protein misfolding is present. Translation errors can cause nonfunctional or toxic proteins, the aggregation of misfolded proteins, and the energy loss of cells. Recent experiments have revealed a relationship between codon optimality and protein folding. Buhr et al. [3] investigated in vivo translation of the recombinant bovine eye-lens protein gamma-B crystallin in E. coli and reported that optimized codons improve the stability and solubility of synthesized proteins. Their NMR spectroscopic data suggested that the translation of optimized codons affects the conformational states of proteins. By performing real-time monitoring in vitro translation system, they also found that optimized codons influence the cotranslational folding of proteins. HK genes might be particularly sensitive to mistranslation because misfolded HK proteins result in the loss of functional molecules in the cells of several tissues. Translational selection that affects the codon usage patterns of HK genes might decrease the energy waste of useless mistranslation or the production of toxic mistranslation products and might provide correct protein products for maintaining cell functions. 


\section{Proportion of protein interacting length and protein connectivity correlate with the CUB of HK genes}

In addition to investigating the possible factors related to CUB at the genomic level, we further investigated CUB of HK genes in relation to the topological properties of HK proteins (proportion of protein interacting length and protein connectivity). We first compared each of these two factors between HK and HTS proteins. HK proteins had a higher percentage of protein interacting length (52.3\%) than did HTS proteins $\left(35.2 \%\right.$, $\mathrm{p}$ value $\left.=6.238 \times 10^{-32}\right)$. In addition, HK proteins had greater protein con-
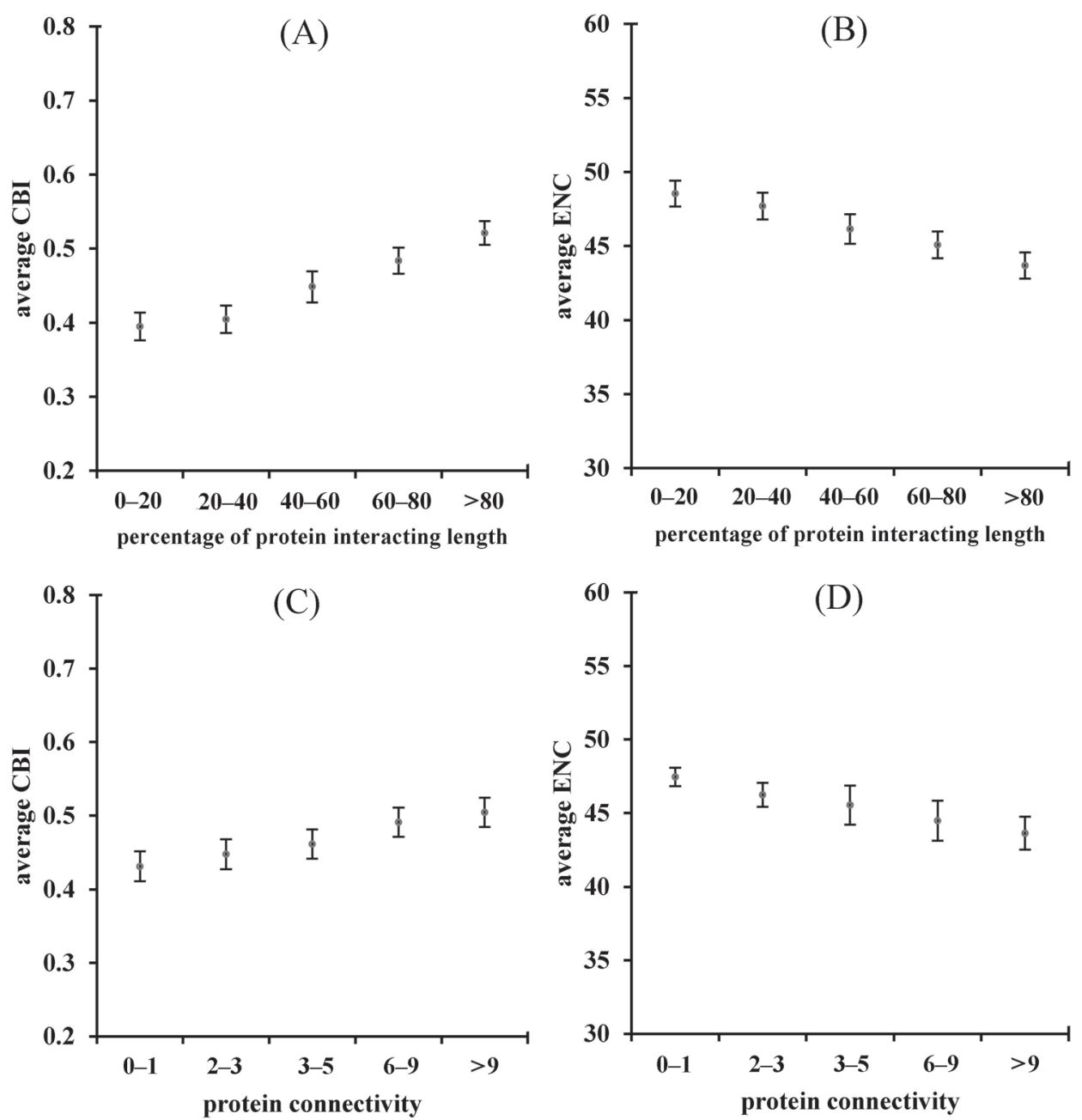

Fig. 2. Scatter Diagrams show correlation between (A) CBI and percentage of protein interacting length

(B) ENC and percentage of protein interacting length (C) CBI and protein connectivity (D) ENC and protein connectivity in HK genes. The error bar shows $95 \%$ confidence interval of the mean 
nectivity $(5.905)$ than did HTS proteins $\left(2.650, \mathrm{p}\right.$ value $\left.=1.439 \times 10^{-60}\right)$. To clarify the relationships between CUB and these two factors, we performed correlation analyses. Table 2 and Fig. 2A-B show that CBI and ENC correlated with the percentage of protein interacting length. What drives the relationship between CUB and percentage of protein interacting length? Zhou et al. [48] reported that in filamentous fungus Neurospora, genome-wide correlations between optimal codons and structured protein domains exist, whereas nonoptimal codons correlate with unstructured protein regions. These correlations were confirmed by in vivo experiments of manipulating the codons for the disordered regions of the Neurospora circadian clock protein FREQUENCY (FRQ). Optimization of codons for the disordered regions of FRQ protein resulted in conformational changes in the FRQ protein. Furthermore, $\mathrm{Fu}$ et al. [16] found that nonoptimal codons correlate with intrinsically disordered regions of Drosophila circadian clock protein (dPER). The optimization of some codons for dPER resulted in changes of protein conformation, phosphorylation profile and stability. Based on previous empirical data and our data, we infer that the percentage of protein interacting length might contribute to synonymous codon usage in HK genes. HK proteins with higher percentages of protein interacting length might be translated by optimal codons that minimize protein misfolding by avoiding mistranslation. Thus, the probability of accurate protein conformation is higher in the case of HK proteins which ensure protein-protein interactions to maintain cellular functions.

The observed correlations between CUB and protein connectivity are shown in Table 2 and Fig. 2C-D. The results indicate that CBI and ENC correlate with protein connectivity. In a previous study, Najafabadi and Salavati [26] suggested that similarity in codon usage is a strong predictor of protein-protein interactions. Dilucca et al. [8] investigated the connection between CUB at the genomic level and topological characteristics of protein-protein interactions in E. coli. They found that hub proteins (proteins with higher numbers of interaction partners) are characterized by higher codon bias of the corresponding genes. Our findings are consistent with the findings in a prokaryote and provide information on the correlation between protein connectivity and CUB in an animal system. What mechanism might explain the relationship between CUB and protein connectivity? It has been reported that proteins with more interaction partners have more constraints on evolutionary rates [15]. The mutation of HK hub proteins might affect protein-protein interaction networks in cells. The biosynthesis of HK hub proteins might occur via optimal codons that decrease the probability of mistranslation and help to achieve accurate protein conformations for interactions. Mistranslated HK hub proteins may lead to protein misfolding and affect protein-protein interactions. By using preferred codons associated with abundant tRNAs, the biosynthesis of HK hub proteins may be more efficient. In contrast, the inefficient translation of $\mathrm{HK}$ hub proteins might reduce the efficiency of protein-protein interactions. The correlation between CUB and the connectivity of HK proteins might facilitate the maintenance of protein functional and physical interactions. 


\section{Rates of nonsynonymous substitution in HK genes are inversely related to $C U B$}

As mentioned above, HK genes have higher CUB than do HTS genes and CUB in $\mathrm{HK}$ genes correlate with four factors $\left(\mathrm{GC}_{3}\right.$ content, gene expression level, proportion of protein interacting length and protein connectivity). In the next set of analyses, we investigated the impact of CUB on the evolution of HK genes. The nonsynonymous substitution rate $(\mathrm{dN})$ in HK and HTS genes was estimated by analyzing $D$. melanogaster $-D$. yakuba orthologs. The average $\mathrm{dN}$ of $\mathrm{HK}$ genes (mean $\mathrm{dN}=0.021$ ) was lower than that of HTS genes (mean $\mathrm{dN}=0.095$, $\mathrm{p}$ value $=2.780 \times 10^{-195}$ ). Differences in dN between HK genes and HTS genes might be mediated by some properties of genes that are correlated with the number of nucleotide substitutions. We then analyzed whether $\mathrm{dN}$ in $\mathrm{HK}$ genes was correlated with CUB. Figure 3A-B show that CBI (Spearman's $\rho=-0.41, \mathrm{p}$ value $\left.=3.973 \times 10^{-46}\right)$ and ENC (Spearman's

Table 3

Spearman's rank correlation analyses between facorts and nonsynonymous substitution rates $(\mathrm{dN})$ in HK genes of $D$. melanogaster

\begin{tabular}{|l|c|c|}
\hline \multicolumn{1}{|c|}{ Factor } & $\rho$ & p value \\
\hline $\mathrm{GC}_{3}$ content & -0.353 & $6.460 \times 10^{-34}$ \\
\hline Expression level & -0.317 & $2.695 \times 10^{-27}$ \\
\hline Percentage of protein interacting length & -0.247 & $9.261 \times 10^{-32}$ \\
\hline Protein connectivity & -0.317 & $2.485 \times 10^{-27}$ \\
\hline
\end{tabular}

Spearman's rank correlation coefficients $(\rho)$ are shown and p values are considered statistically significant.

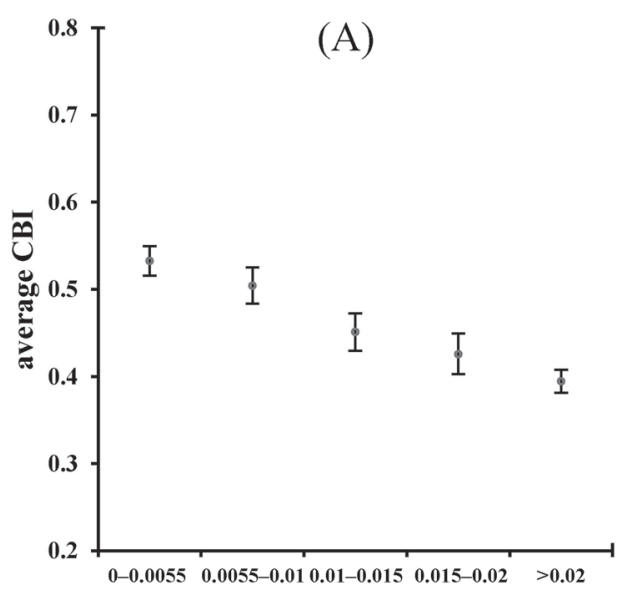

dN

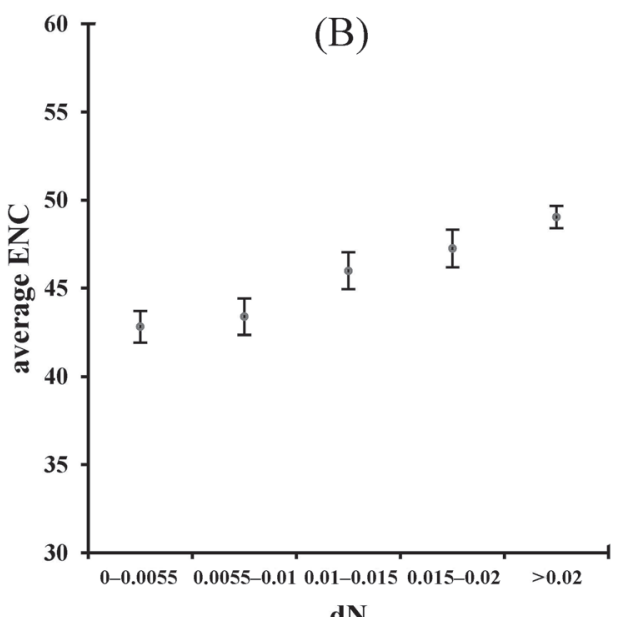

dN

Fig. 3. Scatter Diagrams show correlation between (A) CBI and dN (B) ENC and dN in HK genes. The error bar shows $95 \%$ confidence interval of the mean 
$\rho=0.385, p$ value $=2.033 \times 10^{-40}$ ) were correlated with $\mathrm{dN}$ in HK genes. The correlation between CUB and $\mathrm{dN}$ was also investigated in a previous study. Marais et al. [24] analyzed 630 randomly selected $D$. melanogaster genes (including both high- and low-CUB genes) and found a negative correlation between $\mathrm{dN}$ and the frequency of optimal codons (Fop) in partial genes. A possible explanation for the correlation between CUB and $\mathrm{dN}$ is that nucleotide substitutions resulting in nonoptimal codons might decrease fitness. Previous studies reported that constraints among synonymous codons may slow $\mathrm{dN}$ in some genes $[24,30]$. We found that four factors $\left(\mathrm{GC}_{3}\right.$ content, gene expression level, proportion of protein interacting length and protein connectivity) that correlate with CUB in HK genes also correlate to $\mathrm{dN}$ in HK genes (Table 3). However, the exact cause of the correlation between CUB and dN in HK genes requires further investigation.

\section{CONCLUSIONS}

Based on the results herein, we conclude that differences in CUB exist between the HK genes and HTS genes of D. melanogaster. HK genes have higher CUB than do HTS genes, and CUB correlates with the evolution of HK genes. Mutational bias and natural selection significantly correlate with CUB in the HK genes of D. melanogaster. The proportion of protein interacting length and protein connectivity of HK proteins also correlate with the CUB in the HK genes. This study provides insight into CUB in HK genes of D. melanogaster, and a basis of further investigations for related molecular evolution, genetic engineering and practical applications in insect pest management strategies.

\section{ACKNOWLEDGEMENT}

The authors wish to acknowledge the help of Dr. Chung Yen Lin.

\section{REFERENCES}

1. Bennetzen, J. L., Hall, B. D. (1982) Codon selection in yeast. J. Biol. Chem. 257, 3026-3031.

2. Boutet, E., Lieberherr, D., Tognolli, M., Schneider, M., Bansal, P., Bridge, A. J., Poux, S., Bougueleret, L., Xenarios, I. (2016) UniProtKB/Swiss-Prot, the Manually Annotated Section of the UniProt KnowledgeBase: How to Use the Entry View. Methods Mol. Biol. 1374, $23-54$.

3. Buhr, F., Jha, S., Thommen, M., Mittelstaet, J., Kutz, F., Schwalbe, H., Rodnina, M. V., Komar, A. A. (2016) Synonymous codons direct cotranslational folding toward different protein conformations. Mol. Cell 61, 341-351.

4. Butte, A. J., Dzau, V. J., Glueck, S. B. (2001) Further defining housekeeping, or "maintenance," genes Focus on "A compendium of gene expression in normal human tissues". Physiol. Genomics. 7, 95-96.

5. Carlini, D. B., Stephan, W. (2003) In vivo introduction of unpreferred synonymous codons into the Drosophila Adh gene results in reduced levels of ADH protein. Genetics 163, 239-243.

6. Chaney, J. L., Clark, P. L. (2015) Roles for synonymous codon usage in protein biogenesis. Annu. Rev. Biophys. 44, 143-166. 
7. Crosby, M. A., Goodman, J. L., Strelets, V. B., Zhang, P., Gelbart, W. M. (2007) FlyBase: genomes by the dozen. Nucleic Acids Res. 35, D486-491.

8. Dilucca, M., Cimini, G., Semmoloni, A., Deiana, A., Giansanti, A. (2015) Codon bias patterns of E. coli's interacting proteins. PLoS One 10, 11, e0142127.

9. Drummond, D. A., Wilke, C. O. (2008) Mistranslation-induced protein misfolding as a dominant constraint on coding-sequence evolution. Cell 134, 341-352.

10. Duret, L. (2000) tRNA gene number and codon usage in the C. elegans genome are co-adapted for optimal translation of highly expressed genes. Trends Genet. 16, 287-289.

11. Duret, L. (2002) Evolution of synonymous codon usage in metazoans. Curr. Opin. Genet. Dev. 12, 640-649.

12. Duret, L., Mouchiroud, D. (1999) Expression pattern and, surprisingly, gene length shape codon usage in Caenorhabditis, Drosophila, and Arabidopsis. Proc. Natl Acad. Sci. U S A. 96, 4482-4487.

13. Fath, S., Bauer, A. P., Liss, M., Spriestersbach, A., Maertens, B., Hahn, P., Ludwig, C., Schafer, F., Graf, M., Wagner, R. (2011) Multiparameter RNA and codon optimization: a standardized tool to assess and enhance autologous mammalian gene expression. PLoS One 6, 3 (Mar 03), e17596.

14. Finn, R. D., Coggill, P., Eberhardt, R. Y., Eddy, S. R., Mistry, J., Mitchell, A. L., Potter, S. C., Punta, M., Qureshi, M., Sangrador-Vegas, A., Salazar, G. A., Tate, J., Bateman, A. (2016) The Pfam protein families database: towards a more sustainable future. Nucleic Acids Res. 44, D279-285.

15. Fraser, H. B., Hirsh, A. E., Steinmetz, L. M., Scharfe, C., Feldman, M. W. (2002) Evolutionary rate in the protein interaction network. Science 296, 750-752.

16. Fu, J., Murphy, K. A., Zhou, M., Li, Y. H., Lam, V. H., Tabuloc, C. A., Chiu, J. C., Liu, Y. (2016) Codon usage affects the structure and function of the Drosophila circadian clock protein PERIOD. Genes Dev. 30, 1761-1775.

17. Grantham, R., Gautier, C., Gouy, M., Mercier, R., Pave, A. (1980) Codon catalog usage and the genome hypothesis. Nucleic Acids Res. 8, r49-r62.

18. Gu, W., Zhou, T., Ma, J., Sun, X., Lu, Z. (2004) The relationship between synonymous codon usage and protein structure in Escherichia coli and Homo sapiens. Biosystems 73, 89-97.

19. Hubbell, E., Liu, W. M., Mei, R. (2002) Robust estimators for expression analysis. Bioinformatics 18, $1585-1592$.

20. Kim, P. M., Sboner, A., Xia, Y., Gerstein, M. (2008) The role of disorder in interaction networks: a structural analysis. Mol. Syst. Biol. 4, 179.

21. Knight, R. D., Freeland, S. J., Landweber, L. F. (2001) A simple model based on mutation and selection explains trends in codon and amino-acid usage and GC composition within and across genomes. Genome. Biol. 2, Research0010.

22. Kudla, G., Lipinski, L., Caffin, F., Helwak, A., Zylicz, M. (2006) High guanine and cytosine content increases mRNA levels in mammalian cells. PLoS Biol. 4, e180.

23. Librado, P., Rozas, J. (2009) DnaSP v5: a software for comprehensive analysis of DNA polymorphism data. Bioinformatics 25, 1451-1452.

24. Marais, G., Domazet-Loso, T., Tautz, D., Charlesworth, B. (2004) Correlated evolution of synonymous and nonsynonymous sites in Drosophila. J. Mol. Evol. 59, 771-779.

25. Moriyama, E. N., Powell, J. R. (1998) Gene length and codon usage bias in Drosophila melanogaster, Saccharomyces cerevisiae and Escherichia coli. Nucleic Acids Res. 26, 3188-3193.

26. Najafabadi, H. S., Salavati, R. (2008) Sequence-based prediction of protein-protein interactions by means of codon usage. Genome. Biol. 9, R87.

27. Newman, Z. R., Young, J. M., Ingolia, N. T., Barton, G. M. (2016) Differences in codon bias and GC content contribute to the balanced expression of TLR7 and TLR9. Proc. Natl Acad. Sci. U S A. 113, E1362-1371.

28. Novoa, E. M., Pavon-Eternod, M., Pan, T., Ribas De Pouplana, L. (2012) A role for tRNA modifications in genome structure and codon usage. Cell 149, 202-213.

29. Orchard, S., Ammari, M., Aranda, B., Breuza, L., Briganti, L., Broackes-Carter, F., Campbell, N. H., Chavali, G., Chen, C., Del-Toro, N., Duesbury, M., Dumousseau, M., Galeota, E., Hinz, U., Iannuccelli, M., Jagannathan, S., Jimenez, R., Khadake, J., Lagreid, A., Licata, L., Lovering, R. C., 
Meldal, B., Melidoni, A. N., Milagros, M., Peluso, D., Perfetto, L., Porras, P., Raghunath, A., RicardBlum, S., Roechert, B., Stutz, A., Tognolli, M., Van Roey, K., Cesareni, G., Hermjakob, H. (2014) The MIntAct project-IntAct as a common curation platform for 11 molecular interaction databases. Nucleic Acids Res. 42, D358-363.

30. Pal, C., Papp, B., Hurst, L. D. (2001) Highly expressed genes in yeast evolve slowly. Genetics 158 , 927-931.

31. Pechmann, S., Frydman, J. (2013) Evolutionary conservation of codon optimality reveals hidden signatures of cotranslational folding. Nat. Struct. Mol. Biol. 20, 237-243.

32. Plotkin, J. B., Kudla, G. (2011) Synonymous but not the same: the causes and consequences of codon bias. Nat. Rev. Genet. 12, 32-42.

33. Podder, S., Mukhopadhyay, P., Ghosh, T. C. (2009) Multifunctionality dominantly determines the rate of human housekeeping and tissue specific interacting protein evolution. Gene 439, 11-16.

34. Robinson, S. W., Herzyk, P., Dow, J. A., Leader, D. P. (2013) FlyAtlas: database of gene expression in the tissues of Drosophila melanogaster. Nucleic Acids Res. 41, D744-750.

35. Sharp, P. M., Cowe, E., Higgins, D. G., Shields, D. C., Wolfe, K. H., Wright, F. (1988) Codon usage patterns in Escherichia coli, Bacillus subtilis, Saccharomyces cerevisiae, Schizosaccharomyces pombe, Drosophila melanogaster and Homo sapiens; a review of the considerable within-species diversity. Nucleic Acids Res. 16, 8207-8211.

36. Sharp, P. M., Li, W. H. (1986) An evolutionary perspective on synonymous codon usage in unicellular organisms. J. Mol. Evol. 24, 28-38.

37. Stoletzki, N., Eyre-Walker, A. (2007) Synonymous codon usage in Escherichia coli: selection for translational accuracy. Mol. Biol. Evol. 24, 374-381.

38. Tamura, K., Subramanian, S., Kumar, S. (2004) Temporal patterns of fruit fly (Drosophila) evolution revealed by mutation clocks. Mol. Biol. Evol. 21, 36-44.

39. Vicario, S., Moriyama, E. N., Powell, J. R. (2007) Codon usage in twelve species of Drosophila. BMC Evol. Biol. 7, 226.

40. Waterhouse, R. M., Tegenfeldt, F., Li, J., Zdobnov, E. M., Kriventseva, E. V. (2013) OrthoDB: a hierarchical catalog of animal, fungal and bacterial orthologs. Nucleic Acids Res. 41, D358-365.

41. Weinberg, D. E., Shah, P., Eichhorn, S. W., Hussmann, J. A., Plotkin, J. B., Bartel, D. P. (2016) Improved ribosome-footprint and mRNA measurements provide insights into dynamics and regulation of yeast translation. Cell Rep. 14, 1787-1799.

42. Wright, F. (1990) The 'effective number of codons' used in a gene. Gene 87, 23-29.

43. Yan, X., Hoek, T. A., Vale, R. D., Tanenbaum, M. E. (2016) Dynamics of translation of single mRNA molecules in vivo. Cell 165, 4 (May 05), 976-989.

44. Yanai, I., Benjamin, H., Shmoish, M., Chalifa-Caspi, V., Shklar, M., Ophir, R., Bar-Even, A., HornSaban, S., Safran, M., Domany, E., Lancet, D., Shmueli, O. (2005) Genome-wide midrange transcription profiles reveal expression level relationships in human tissue specification. Bioinformatics 21 , 650-659.

45. Yang, Z. (1997) PAML: a program package for phylogenetic analysis by maximum likelihood. Comput. Appl. Biosci. 13, 555-556.

46. Yang, Z., Nielsen, R. (2000) Estimating synonymous and nonsynonymous substitution rates under realistic evolutionary models. Mol. Biol. Evol. 17, 32-43.

47. Zamft, B., Bintu, L., Ishibashi, T., Bustamante, C. (2012) Nascent RNA structure modulates the transcriptional dynamics of RNA polymerases. Proc. Natl Acad. Sci. U S A. 109, 8948-8953.

48. Zhou, M., Guo, J., Cha, J., Chae, M., Chen, S., Barral, J. M., Sachs, M. S., Liu, Y. (2013) Non-optimal codon usage affects expression, structure and function of clock protein FRQ. Nature 495, 111-115.

49. Zhou, T., Gu, W., Ma, J., Sun, X., Lu, Z. (2005) Analysis of synonymous codon usage in H5N1 virus and other influenza A viruses. Biosystems 81, 77-86.

50. Zhou, T., Weems, M., Wilke, C. O. (2009) Translationally optimal codons associate with structurally sensitive sites in proteins. Mol. Biol. Evol. 26, 1571-1580. 